\title{
Caregivers' nutrition knowledge and attitudes are associated with household food diversity and children's animal source food intake across different agro-ecological zones in Ghana
}

\author{
Aaron K. Christian ${ }^{1 *}$, Grace S. Marquis ${ }^{2,3}$, Esi K. Colecraft ${ }^{4}$, Anna Lartey ${ }^{4}$, Owuraku Sakyi-Dawson ${ }^{5}$, \\ Ben K. Ahunu ${ }^{6}$ and Lorna M. Butler ${ }^{7}$ \\ ${ }^{1}$ Regional Institute for Population Studies, University of Ghana, PO Box LG96, Legon, Ghana \\ ${ }^{2}$ School of Dietetics and Human Nutrition, McGill University, Ste. Anne-de-Bellevue, QC H9X 3V9, Canada \\ ${ }^{3}$ Departments of Food Science and Human Nutrition, Iowa State University, 2312 Food Sciences Building, Ames, IA 50011-1061, USA \\ ${ }^{4}$ Department of Nutrition and Food Science, University of Ghana, PO Box LG 134, Legon, Ghana \\ ${ }^{5}$ Department of Agricultural Extension, University of Ghana, Legon, Ghana \\ ${ }^{6}$ Department of Animal Science, College of Agriculture and Consumer Sciences, University of Ghana, Legon, Ghana \\ ${ }^{7}$ Department of Sociology, Iowa State University, North Saanich, BC, Canada
}

(Submitted 5 January 2015 - Final revision received 13 September 2015 - Accepted 12 October 2015 - First published online 12 November 2015)

\begin{abstract}
Caregivers' nutrition knowledge and attitudes may influence the variety of foods available in the household and the quality of children's diets. To test the link, this study collected data on caregivers' ( $n$ 608) nutrition knowledge and feeding attitudes as well as the diets of their household and of their 2-5-year-old children in twelve rural communities nested in the three main agro-ecological zones of Ghana. Household foods and children's animal source foods (ASF) consumed in the past $7 \mathrm{~d}$ were categorised into one of fourteen and ten groups, respectively. About $28 \%$ of caregivers believed that their children needed to be fed only $2-3$ times/d. Reasons for having adult supervision during child meal times, feeding diverse foods, prioritising a child to receive ASF and the perceived child benefits of ASF differed across zones $(P<0 \cdot 001)$. Households with caregivers belonging to the highest tertile of nutrition knowledge and attitude scores consumed more diverse diets compared with those of caregivers in the lowest tertile group (11.2 (sD 2.2) v.10.0 (sD 2.4); P<0.001). After controlling for the effect of agro-ecological zone, caregivers' nutrition knowledge and feeding attitudes positively predicted household dietary diversity and the frequency and diversity of children's ASF intakes $(P<0 \cdot 001)$. The number of years of formal education of caregivers also positively predicted household dietary diversity and children's ASF diversity $(P<0 \cdot 001)$. A key component to improving child nutrition is to understand the context-specific nutrition knowledge and feeding attitudes in order to identify relevant interventions.
\end{abstract}

Key words: Caregivers' nutrition knowledge: Household dietary diversity: Animal source foods: Agro-ecological zones

Poor childhood nutritional status in sub-Saharan Africa continues to be a challenge and major risk factor for disability and premature mortality ${ }^{(1-4)}$. The UNICEF model and other operational frameworks show the importance of overall child care practices, including interactive behavioural processes between caregivers and children at meal times, to influence child nutrition and health ${ }^{(5,6)}$. The feeding behaviour of caregivers includes decisions about how, why, when and what they feed their children. Central to the recommendations for responsive feeding is ensuring the availability of healthy foods and providing a comfortable feeding environment for the child $^{(7)}$. There is ample evidence that more diverse meals are healthier than meals consisting of a single food item or fewer food groups ${ }^{(8)}$. The consumption of a diverse diet, particularly one that includes animal source foods (ASF) ${ }^{(9)}$, can help meet children's nutritional requirements ${ }^{(10)}$ and has been found to be protective against stunting and underweight ${ }^{(11-13)}$. Although consumption of ASF in general is encouraged, specific foods have a unique contribution to child growth and development and ASF diversity is important ${ }^{(14)}$. The present study, therefore, focused on ASF diversity.

The type, quantity and diversity of food that is chosen for a child may be determined by household and individual characteristics interacting in complex ways to influence the development and maintenance of caregivers' nutrition knowledge and attitudes. Understanding the dietary choices

Abbreviations: ASF, animal source food; ENAM, Enhancing Child Nutrition through Animal Source Food Management.

* Corresponding author: A. K. Christian, email christiankobina@yahoo.com 
and caregivers' reasons for those choices will aid in formulating guidelines to improve child feeding practices ${ }^{(15)}$. To date, there is a paucity of information in the literature on caregivers' nutrition knowledge and attitudes, particularly concerning children's intake of ASF.

The present study was carried out in Ghana, a West African country that relies mainly on rain-fed agriculture, and thus is vulnerable to food insecurity. Children's diets consist primarily of cereals, roots and tubers with little or no ASF; the poor quality of the diet contributes to the high prevalence of micronutrient deficiencies $^{(16-18)}$. The 2008 Ghana Demographic and Health Survey estimated that $28 \%$ of children under 5 years of age were stunted with high variation across agro-ecological zones ${ }^{(19)}$. To address this public health concern, the Enhancing Child Nutrition through Animal Source Food Management (ENAM) project developed a context-specific problem model for the causal links to low ASF consumption among young children in rural Ghana. The resulting intervention integrated microcredit with nutrition and entrepreneurial education. The ENAM project methods of assessment, problem model, intervention priorities as well as growth outcomes have been published previously ${ }^{(20,21)}$.

To guide the nutrition education intervention, the project collected data on (i) the diets of households and their 2-5year-old children and (ii) caregivers' nutrition knowledge and attitudes, especially about ASF, and compared these with standard dietary recommendations and infant and young children feeding recommendations ${ }^{(22,23)}$. We hypothesised that caregivers' nutrition knowledge and attitudes independently were associated with household dietary diversity and children's ASF consumption.

\section{Methods}

\section{Study sites and participants}

The data used for this analysis were collected as baseline information for the ENAM project between April 2006 and February 2007. The peak rainy season and the driest months correspond to April to July and November to February, respectively. In consultation with staff from the Ghanaian Ministry of Food and Agriculture, three different agro-ecological zones (Guinea Savannah Zone, Forest Transitional Zone and Coastal Savannah Zone) were purposively selected to reflect the diversity of ASF across the country. The survey procedures for this study have been described in detail previously ${ }^{(20,21)}$.

An exhaustive list of 608 caregivers with their 2-5-year-old children were identified through community mapping and included all mothers who were willing to participate in the survey and be followed-up for successive data collection for the ENAM study. A caregiver was defined as the person with whom the child lived and who had primary responsibility for child care (including feeding) most of the time. For households with caregivers having more than one child within the eligible age, the youngest child was selected as the index child participant.

\section{Data collection}

Research staff were trained to administer the study questionnaire in the language preferred by the participant. The questions were pretested in neighbouring communities and revised as needed. Data were collected through in-person interviews with the caregivers in their homes. Field supervisors randomly duplicated questionnaires to cross-check answers recorded by field staff. Screening questions were incorporated into the questionnaire to identify ineligible respondents and to check the respondent's credibility.

Household characteristics. Caregivers reported on socioeconomic and demographic characteristics, including household composition and the participant's education and occupation. A sex-balanced key informant group was set up in each community to assign a wealth rank to the selected study households ${ }^{(24)}$. Key informants defined for their own community criteria for a household to be considered as having high, medium or low wealth - for example, across study communities, high wealth status was assigned if a family member, particularly the household head, had a profession and regular income, or was able loan money to others. A household ranked as having medium wealth was perceived as not being too rich but able to send their children to school. Criteria used to rank a household as having low wealth included the inability to send their children to school and dependence only on farming for income. A dichotomous variable was used for wealth rank $(0=$ low $; 1=$ medium or high $)$.

Caregivers' nutrition knowledge and attitudes. Field staff asked caregivers about their nutrition knowledge and attitudes about specific feeding practices, as well as the reasons for their responses (Table 1). Correct answers and correct reasons were scored 1 and 2, respectively. Incorrect answers and incorrect reasons or missing values were scored 0 . The sum of the scores from the questions was calculated to give a maximum score of 14. The overall nutrition knowledge and attitudes score was divided into tertiles. Caregivers in the third tertile had the highest nutrition knowledge and attitudes score.

Dietary intake. The FFQ was developed based on the guidelines from the Nutrition and Consumer Protection Division of the Food and Agriculture Organization ${ }^{(25,26)}$. Caregivers were interviewed about foods consumed in their households in the past $7 \mathrm{~d}$. The questionnaire included a list of commonly consumed foods categorised into fourteen food groups (roots and tubers, cereals, legumes, seeds and nuts, red meat, poultry, eggs, fish and seafood, dairy products, green leafy vegetables, other vegetables, citrus fruits, other fruits and fats/oils).

Using another FFQ, caregivers were asked to recall all ASF consumed by their children over the past $7 \mathrm{~d}$. The ten categories included the following: livestock meats (pork, goat, mutton and beef), organ meats and offal, game animals, whole fish, fish powder, shellfish, snails, poultry (chicken, duck, guinea fowl), eggs and milk and milk products ${ }^{(21,27)}$. Children's ASF frequency was calculated as the number of times a child consumed an ASF over the past $7 \mathrm{~d}$. ASF diversity was estimated as the number of different ASF categories consumed within the same time reference. 
Table 1. Caregivers' nutrition knowledge and attitudes questions, response examples and scoring scheme

\begin{tabular}{|c|c|c|c|}
\hline & Questions & Response examples* & Score \\
\hline \multirow[t]{2}{*}{1} & How many times a day should a $2-5$-year-old child eat in order to grow well and be healthy? & $\geq 3$ times/d & 1 \\
\hline & & $<3$ times/d & 0 \\
\hline \multirow[t]{2}{*}{2} & Is it necessary for a $2-5$-year-old child to be given snacks in between main meals? & Yes & 1 \\
\hline & & No & 0 \\
\hline \multirow[t]{2}{*}{3} & Is it necessary for an adult to watch over a $2-5$-year-old child while he/she eats? & Yes & 1 \\
\hline & & No. The child will eat what he/she will eat & 0 \\
\hline 4 & If yes to Question 3, ask why? & $\begin{array}{l}\text { To attend to child needs and encourage } \\
\text { feeding }\end{array}$ & 2 \\
\hline \multirow[t]{2}{*}{5} & If there is only a small amount of ASF in the evening meal to be shared among household & Parents or adults in the household & 0 \\
\hline & & My 2-5-year-old child & 2 \\
\hline \multirow[t]{2}{*}{6} & Why your answer for Question 5 & Because the adult need the ASF more & 0 \\
\hline & & Because children need ASF more & 2 \\
\hline \multirow[t]{2}{*}{7} & Is it important that mothers give their $2-5$-year-old children different types of food to eat? & Yes & 1 \\
\hline & & $\begin{array}{l}\text { Not necessary, as long as his/her stomach is } \\
\text { full }\end{array}$ & 0 \\
\hline \multirow[t]{2}{*}{8} & If yes to Question 7, ask why? & Different foods have different taste & 0 \\
\hline & & $\begin{array}{l}\text { Different food provide different nutrients } \\
\text { necessary for growth }\end{array}$ & 2 \\
\hline \multirow[t]{2}{*}{9} & Is important for mothers to try their best to include ASF into their children meals & No & 0 \\
\hline & & Yes & 1 \\
\hline \multirow[t]{2}{*}{10} & If yes to Question 9, ask why? & Only because it is tasty & 0 \\
\hline & & $\begin{array}{l}\text { ASF contains nutrient necessary for optimum } \\
\text { growth }\end{array}$ & 2 \\
\hline
\end{tabular}

ASF, animal source foods.

* These were open-ended responses. Credit was given when the response reflected the nutrition concept shown in the table.

\section{Data management and statistical analysis}

The data were analysed using the Statistical Package for Social Scientists version 19 (SPSS Inc.) and Stata version $12^{(28)}$. Table 1 lists the questions used to assess caregivers' nutrition knowledge and attitudes, typical responses and scores assigned to the responses. Total nutrition knowledge and attitudes score (range 1-14) was calculated from the sum of the values for each question. The knowledge and attitudes scores were categorised into tertiles, and the means of the dietary intakes were compared across the three levels. The continuous knowledge and attitudes score was used for the multilevel analysis.

A household dietary diversity score was constructed from the fourteen food groups. A value of 0 was given to a food group if no household member consumed any item in that food group in the past $7 \mathrm{~d}$; a value of 1 was given if a member consumed something in the food group. The dietary diversity score had a maximum score of 14 (eating from all fourteen food groups). Child ASF diversity score was defined as the number of different ASF groups a child consumed at least once in the past $7 \mathrm{~d}$. The minimum score for child ASF diversity was 0 (did not consume any form of ASF) and the maximum was 10 (consumed from all ten groups of ASF).

The primary outcomes of the current analysis were household dietary diversity, child ASF diversity and child ASF frequency. Caregivers' nutrition knowledge and attitudes score, characteristics of the household (wealth and size) and caregiver (age and educational status) and agro-ecological zones were the independent variables. Study variables were described using frequencies as well as means and standard deviations. Associations were first examined using bivariate analysis.
Post-hoc tests (Bonferroni) were conducted for differences in means for variables with more than two categories.

\section{Multilevel analysis}

Given the hierarchical nature of the sampling design, a multilevel model was used to explore the relationship between caregivers' nutrition knowledge and attitudes and the three outcomes of interest across the different agro-ecological zones. Individual caregiver's characteristics that were entered in the model included the following: nutrition knowledge and attitudes, number of years of formal education, employment status, type of income-generation activity (whether or not it was related to ASF) and household wealth rank. Community characteristics that potentially influenced the outcomes of dietary intake included the mean proportion of food items purchased within the community. This variable was used as a proxy for the food environment index of the community ${ }^{(29)}$ and treated as a random effect.

The agro-ecological zones served as level-2 information in the multilevel analysis. Using multilevel modelling helps overcome the problem of disregarding potential grouping effects ${ }^{(30)}$. These effects often lead to miscalculating the standard errors of regression coefficients by isolating ecological-specific effects from the effects of other covariates, thus taking the contextual influences into account ${ }^{(31)}$. We centred values of predictor variables to reduce non-essential collinearity between interaction terms and their components. Models were tested against the null model. Statistical significance was tested at $P<0.05$. The multilevel model fit was assessed using likelihood ratio tests in Stata statistical package version 12. 


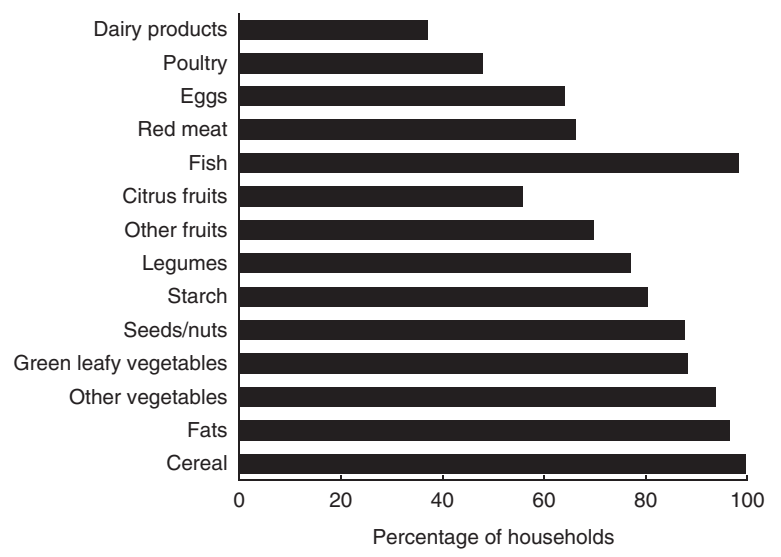

Fig. 1. Percentage of household who consumed from each food group during the past $7 \mathrm{~d}$.

\section{Ethical considerations}

The study was approved by the Institutional Review Boards of Iowa State University, the Noguchi Memorial Institute for Medical Research at the University of Ghana, Legon, and McGill University. All the participants provided written informed consent for themselves and their children.

\section{Results}

\section{Background characteristics of study participants}

Almost two-thirds (61.0\%) of the participating households were ranked as having low wealth status. The mean age of the caregivers was 32.8 (sD 8.9 ) years, $12.8 \%$ were single (either never married, divorced or widowed) and more than half $(52 \cdot 1 \%)$ of them had received no formal education. The mean height-for-age, weight-for-age and weight-for-height $Z$-scores for children were $-1.4(\mathrm{SD} 1.2),-0.9$ (SD 1.0) and -0.2 (SD 0.9), respectively. On the basis of the World Health Organization child growth standards, $28.0,13.2$ and $2.0 \%$ of the children were stunted, underweight and wasted, respectively.

\section{Dietary intake}

Household dietary diversity. The household dietary diversity score ranged from 4 to 14 with a mean of $10 \cdot 6$ (SD 2.4) food groups. Fig. 1 shows the proportion of households that consumed the different food groups at least once in the past $7 \mathrm{~d}$. Almost all households (99.7\%) reported consuming cereals; this was followed by fats and oil (96.5\%), non-green leafy vegetables $(93.8 \%)$, green leafy vegetables $(88.3 \%)$ and nuts and seeds (87.7\%). The most common animal food group consumed was fish (98.4\%). Less than half of the households had consumed poultry $(47.9 \%)$ or dairy products $(37 \cdot 2 \%)$.

Household dietary diversity differed significantly $(P<0 \cdot 001)$ across the zones (Table 2). In comparison with the Forest Transition zone and Coastal Savannah zone, households in the Guinea Savannah had the least diverse diets. It also differed significantly across different wealth-ranked households, with higher diversity among households ranked medium/high.

Children's animal source food diversity and frequency. The intake of ASF varied widely among children; the ASF diversity score ranged from 0 to 10 with a mean of 4.7 (SD 2.1) foods. The frequency of ASF consumption within the past $7 \mathrm{~d}$ varied from 0 to 24 times with a mean of $10 \cdot 2$ (SD 4.7). About $90 \%(89.6 \%)$ of children consumed whole fish, making it the most frequently consumed ASF (Fig. 2). This was followed by fish powder $(71.5 \%)$ and eggs $(71.2 \%)$. The least consumed types of ASF were bushmeats and snails (26.6 and $21.5 \%$, respectively). Children's ASF diversity and frequency differed significantly $(P<0.0001)$ across the zones, with children living in the Forest Transitional zone consuming the most diverse ASF compared with children in the Guinea Savannah zone and Coastal Savannah zone. It also differed by wealth rank, with the higher intake of both indicators among households that ranked medium/high.

\section{Caregivers' nutrition knowledge and attitudes}

When asked about appropriate meal frequency, the majority of caregivers $(71 \cdot 1 \%)$ believed that their 2-5-year-old children needed to be fed $>3$ times/d (Table 3 ). Over $80 \%$ of caregivers considered snacks to be an important component of their children's diet. Although almost $90 \%$ of caregivers believed it necessary to supervise children's meal times, only $50 \%$ of them were able to articulate a correct reason for the need for supervision, with significantly fewer caregivers from the Guinea Savannah zone compared with caregivers in the Forest Transition and Coastal Savannah zone providing accurate reasons $(P<0 \cdot 001)$. Caregivers were asked to indicate who in their household they would prioritise if there were limited ASF in a household meal. About two-thirds of them said they would prioritise giving the scarce ASF to children, irrespective of the child's sex; however, the remaining (33.9\%) indicated that they would give it to other household members. Among those who said they would prioritise children, $20 \%$ fewer caregivers in the Coastal Savannah zone compared with the other two zones $(P<0.05)$ gave good/correct reasons for the need to prioritise children. Over $90 \%$ of caregivers believed it was necessary to feed children diverse diets and $98 \%$ said ASF was important. The highest percentage of correct reasons for these two behaviours was among caregivers in the Forest Transition zone. The lowest tertile for the nutrition knowledge and attitudes score had the lowest mean scores for household diversity, child ASF diversity and child ASF frequency. The scores were significantly different from those of the highest tertile category (Table 4).

Multilevel analysis. To determine the extent to which the agro-ecological zone differences explained individual household-level variation in diversity scores, we fit the data to a series of random intercept models (Table 5). For household dietary diversity, the null model (model 1 ) showed that the mean score was $10 \cdot 7$. The intra-class correlation coefficient 
Table 2. Household dietary diversity and child animal source food (ASF) diversity and frequency scores, by socio-economic and demographic characteristics (Numbers, mean values and standard deviations)

\begin{tabular}{|c|c|c|c|c|c|c|c|}
\hline \multirow[b]{2}{*}{ Characteristics } & \multirow[b]{2}{*}{$n$} & \multicolumn{2}{|c|}{ Household dietary diversity* } & \multicolumn{2}{|c|}{ Child ASF diversity† } & \multicolumn{2}{|c|}{ Child ASF frequency } \\
\hline & & Mean & SD & Mean & SD & Mean & SD \\
\hline \multicolumn{8}{|l|}{ Agro-ecological zone } \\
\hline Coastal Savannah & 131 & $10 \cdot 9^{a}$ & 2.4 & $4 \cdot 7^{\mathrm{a}}$ & 1.8 & $11 \cdot 1^{\mathrm{a}}$ & 4.7 \\
\hline Forest Transitional & 235 & $11 \cdot 8^{b}$ & $2 \cdot 0$ & $6.0^{\mathrm{b}}$ & $2 \cdot 3$ & $12 \cdot 2^{b}$ & 4.8 \\
\hline Guinea Savannah & 242 & $9 \cdot 3^{\mathrm{c}}$ & $2 \cdot 0$ & $3 \cdot 6^{\mathrm{c}}$ & $1 \cdot 4$ & $7 \cdot 8^{\mathrm{C}}$ & 3.2 \\
\hline \multicolumn{8}{|l|}{ Household wealth } \\
\hline Low & 369 & $10 \cdot 4^{\mathrm{a}}$ & $2 \cdot 4$ & $4 \cdot 5^{\mathrm{a}}$ & $2 \cdot 1$ & $9 \cdot 6^{\mathrm{a}}$ & 4.5 \\
\hline Medium/high & 239 & $11 \cdot 0^{\mathrm{b}}$ & $2 \cdot 3$ & $5 \cdot 2^{\mathrm{b}}$ & $2 \cdot 1$ & $11 \cdot 2^{b}$ & 4.8 \\
\hline \multicolumn{8}{|l|}{ Household head } \\
\hline Female & 128 & $10 \cdot 9$ & $2 \cdot 4$ & 4.9 & $2 \cdot 0$ & $10 \cdot 6$ & 4.7 \\
\hline Male & 408 & 10.5 & $2 \cdot 4$ & 4.8 & $2 \cdot 2$ & $10 \cdot 1$ & 4.7 \\
\hline \multicolumn{8}{|l|}{ Caregiver age (years) } \\
\hline$<25$ & 101 & $10 \cdot 3$ & $2 \cdot 7$ & 4.6 & $2 \cdot 3$ & 9.9 & $5 \cdot 0$ \\
\hline $25-29$ & 125 & 10.9 & $2 \cdot 2$ & 4.9 & $2 \cdot 0$ & 10.4 & 4.6 \\
\hline $30-39$ & 248 & $10 \cdot 7$ & $2 \cdot 2$ & 4.9 & $2 \cdot 2$ & $10 \cdot 3$ & 4.5 \\
\hline $40-49$ & 93 & 10.5 & 2.6 & 4.6 & $2 \cdot 3$ & 9.9 & $5 \cdot 1$ \\
\hline$\geq 50$ & 41 & $10 \cdot 0$ & $2 \cdot 5$ & 4.6 & $2 \cdot 2$ & $9 \cdot 8$ & 4.4 \\
\hline \multicolumn{8}{|l|}{ Marital status } \\
\hline Single & 78 & $10 \cdot 3$ & $2 \cdot 5$ & 4.4 & $2 \cdot 1$ & 9.7 & 4.9 \\
\hline Married & 530 & $10 \cdot 7$ & $2 \cdot 4$ & 4.8 & $2 \cdot 2$ & $10 \cdot 3$ & 4.7 \\
\hline \multicolumn{8}{|l|}{ Caregiver education } \\
\hline None & 317 & $10 \cdot 1^{\mathrm{a}}$ & 2.5 & $4 \cdot 4^{\mathrm{a}}$ & $2 \cdot 1$ & $9 \cdot 6^{\mathrm{a}}$ & 4.6 \\
\hline Primary & 135 & $10 \cdot 6^{b}$ & $2 \cdot 1$ & $4 \cdot 6^{\mathrm{a}}$ & 1.9 & $9 \cdot 8^{\mathrm{a}}$ & 4.3 \\
\hline Secondary or more & 156 & $11 \cdot 7^{\mathrm{c}}$ & $2 \cdot 0$ & $5 \cdot 6^{\mathrm{b}}$ & $2 \cdot 3$ & $11 \cdot 8^{b}$ & 4.9 \\
\hline
\end{tabular}

a,b,c Mean values within a column with unlike superscript letters were significantly different (the Bonferroni test was use for multiple-comparison; $P<0.05$ ).

* Summative score for roots and tubers, cereals, legumes, seeds and nuts, red meat, poultry, eggs, fish and seafood, dairy products, green leafy vegetables, other vegetables, citrus fruits, other fruits and fats/oils consumed the past week (range: 4-14).

† Summative score for livestock meats, organ meats and offal, bushmeats, whole fish, fish powder, shellfish, snails, poultry, eggs and milk and milk products consumed the past week (range: $0-10$ ).

‡ Summative score of frequency for the ASF consumed the past week (range: $0-24$ ).

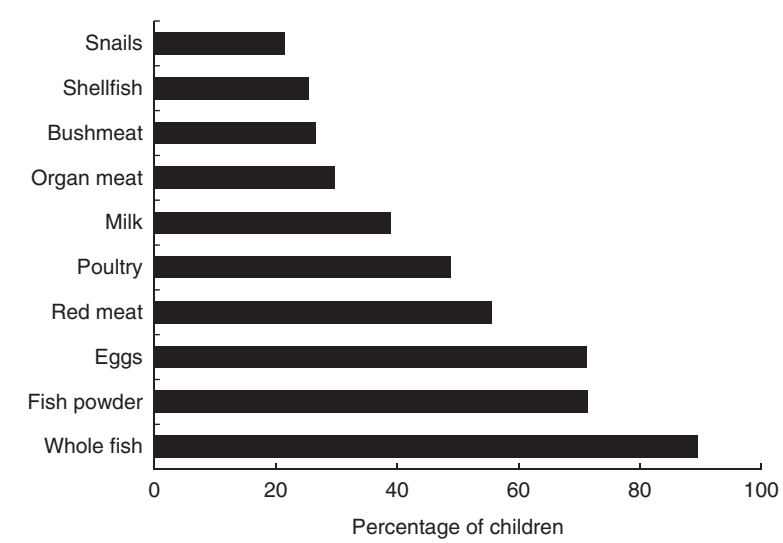

Fig. 2. Percentage of children who consumed at least once from different animal source food group.

(ICC) of 0.20 in model 1 suggested that $20 \%$ of variance in a household's dietary diversity was attributable to agro-ecological zone level, justifying the use of a multilevel model analysis.

This contextual effect was modified significantly $(P<0 \cdot 001)$ by the introduction of caregivers' characteristics (model 2). Household dietary diversity increased with a higher number of years of formal education of caregivers and a better nutrition knowledge and attitudes score. Having an employed caregiver also increased the household diversity score. The variance component corresponding to the random intercept decreased, reflecting that the inclusion of level- 2 variables accounted for some of the variance in the dependent variable. These effects, although statistically significant, were very modest. The effect of household wealth and the sex of the household head and their interaction terms were explored and found not to contribute to the model; their interaction was also not significant and excluded from the model.

A final model (model 3) introduced an index for the food environment of the community. The effect of the food environment on the dietary intake may vary across communities; thus, this slope was treated as random. Household dietary diversity increased with an increase in the mean proportion of needed food items purchased within a community. In other words, living in a community with higher availability of needed foods increased the dependent outcome. The variance component for the random intercept was $0 \cdot 807$, which is still large relative to its SE of 0.397 . Thus, there remains still some unaccounted variance. However, comparing the Akaike information criterion (AIC) and Bayesian information criterion (BIC) statistics in Table 5 it is clear that the final model is preferred to the first two models.

The models for children's ASF intake were similar to that of household diet diversity (Table 5). Model 1 for child ASF diversity indicated that the mean ASF diversity was 4.8 foods. The ICC for the model was $0 \cdot 333$, meaning that about $33 \%$ of total variance in diversity was attributable to agro-ecological zone level. Model 2 was used to further determine the influence of caregiver and 
Table 3. Proportion of caregivers with correct child nutrition knowledge and attitudes, by agro-ecological zones (Percentages)

\begin{tabular}{|c|c|c|c|c|}
\hline \multirow[b]{2}{*}{ Nutrition knowledge and attitudes } & \multicolumn{3}{|c|}{ Agro-ecological zones } & \multirow[b]{2}{*}{$P^{*}$} \\
\hline & Coastal Savannah ( $n$ 131) & Forest Transition ( $n$ 235) & Guinea Savannah ( $n$ 242) & \\
\hline Meal frequency $>3$ times $/ d$ & $70 \cdot 2$ & $67 \cdot 2$ & $76 \cdot 9$ & 0.060 \\
\hline Snacks are important & $87 \cdot 0$ & 91.9 & 85.5 & 0.083 \\
\hline Meal supervision is important & 91.6 & 89.8 & 88.4 & 0.628 \\
\hline Correct reason for supervising meal† & $51 \cdot 7$ & 61.6 & $36 \cdot 0$ & $<0.001$ \\
\hline ASF prioritised to child, regardless of sex & $71 \cdot 0$ & 48.5 & $80 \cdot 6$ & 0.032 \\
\hline Correct reason for prioritising ASF for child & $52 \cdot 7$ & $66 \cdot 7$ & $66 \cdot 7$ & $<0.001$ \\
\hline Dietary diversity is necessary & 98.5 & $98 \cdot 7$ & $95 \cdot 0$ & 0.032 \\
\hline Correct reason for why diversity is needed $\ddagger$ & 91.5 & 93.5 & $86 \cdot 1$ & 0.001 \\
\hline ASF is important & 98.5 & 98.3 & $96 \cdot 7$ & 0.405 \\
\hline Correct reason for why is ASF important§ & $83 \cdot 7$ & 91.3 & $78 \cdot 2$ & $<0.001$ \\
\hline
\end{tabular}

ASF, animal source food.

* Comparison of agro-ecological groups, using $\chi^{2}$ goodness-of-fit test.

† Total sample size was 545: Coastal Savannah 120, Forest Transition 211 and Guinea Savannah 214

‡ Sample size was 591: Coastal Savannah 129, Forest Transition 232 and Guinea Savannah 230.

§ Sample size was 594: Coastal Savannah 129, Forest Transition 230 and Guinea Savannah 234.

Table 4. Association between caregivers' knowledge and attitudes score and dietary intake (Mean values and standard deviations)

\begin{tabular}{|c|c|c|c|c|c|c|}
\hline \multirow[b]{3}{*}{ Dietary intakes } & \multicolumn{6}{|c|}{ Tertile category of caregiver's nutrition knowledge and attitudes score } \\
\hline & \multicolumn{2}{|c|}{ Low $(n$ 226) } & \multicolumn{2}{|c|}{ Medium ( $n$ 259) } & \multicolumn{2}{|c|}{ High (n 123) } \\
\hline & Mean & SD & Mean & SD & Mean & SD \\
\hline Household dietary diversity & $10 \cdot 0^{\mathrm{a}}$ & $2 \cdot 4$ & $10.9^{\mathrm{b}}$ & $2 \cdot 3$ & $11 \cdot 2^{\mathrm{b}}$ & $2 \cdot 2$ \\
\hline Child ASF diversity & $4 \cdot 4^{\mathrm{a}}$ & $2 \cdot 1$ & $5.0^{\mathrm{b}}$ & $2 \cdot 3$ & $5 \cdot 2^{\mathrm{b}}$ & $2 \cdot 0$ \\
\hline Child ASF frequency & $9 \cdot 3^{\mathrm{a}}$ & 4.4 & $10 \cdot 6^{\mathrm{b}}$ & 4.9 & $11 \cdot 3^{\mathrm{b}}$ & 4.4 \\
\hline
\end{tabular}

ASF, animal source food.

a,b,c Mean values within a row with unlike superscript letters were significantly different (the Bonferroni test was used for multiple comparisons; $P<0.05$ ).

household characteristics. Child ASF diversity increased with caregivers' nutrition knowledge and attitudes score and having a caregiver engaged in an ASF-related work. Household wealth, sex of the household head and household size and their interaction terms were also explored and found not to contribute to the model.

The multilevel modelling result for child ASF frequency followed a similar direction as that for ASF diversity. However, household wealth predicted ASF frequency. Compared with children in low wealth-ranked households, children in medium/high wealth-ranked households consumed ASF more frequently. The progressive increase in the log-likelihood observed in model 2 and model 3 and also importantly the decrease in AIC values indicated that the latter models better explained the determinants.

\section{Discussion}

After controlling for the effect of agro-ecological zone, caregivers' nutrition knowledge and feeding attitudes positively predicted both household dietary diversity and children's ASF diversity and ASF frequency. The multilevel analysis model allowed us to account for inequalities that existed in household food diversity and children's ASF diversity and frequency attributable to the three agro-ecological zones, thus addressing adequately the hierarchical structure of the data. The present study was unique in that it examined nutrition knowledge and attitudes concerning specific feeding practices, particularly about ASF, and the reasons behind some feeding attitudes.

These findings extend previous research in several important ways. First, these results go beyond previous research that have studied the association of caregivers' nutrition knowledge and attitudes with certain child outcomes and provide insights into specific reasons for feeding practices. This study suggested the need to equip caregivers with more knowledge concerning specific feeding recommendations to ensure better adherence to nutritional guidelines. About one-third of caregivers said they would prioritise other household members over children when there is limited ASF, pointing to the continued existence of some cultural beliefs and practices that are detrimental to the feeding of young children.

These findings corroborate those from earlier studies conducted among a similar socio-economic class in Ghana. Maternal knowledge gained through informal education moderated the negative consequences of poverty and low maternal schooling level on indicators of nutritional status ${ }^{(32)}$. Moreover, the ability of mothers to adhere to recommended child feeding practices has been shown to be linked to maternal nutritional knowledge ${ }^{(33)}$. 
Nutrition knowledge and household food intake

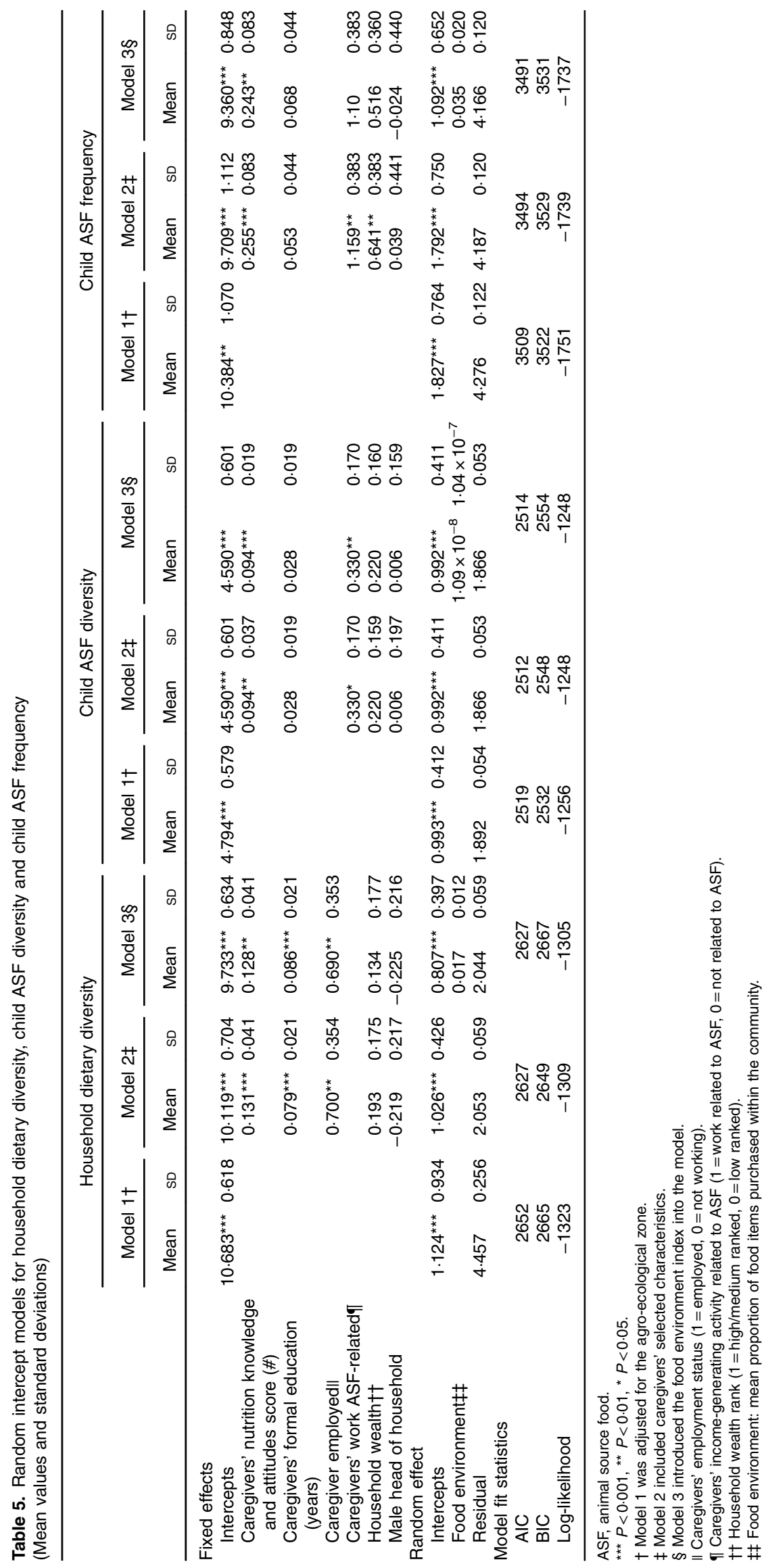


Results of the multilevel analysis showed that within each agro-ecological zone, the dietary outcomes were more strongly associated with caregivers' nutrition knowledge and attitudes than with wealth. Although ample attention has been given to socio-demographic characteristics, this study emphasised the need to recognise the complex interplay of factors that influence what foods households eat and particularly what children are fed. Blaylock et $a l .{ }^{(34)}$ pointed out that 'consumers make dietary decisions based on economic, physiologic, psychological, sociologic and even spiritual considerations'.

The positive association between the caregiver's formal education and household dietary diversity has been observed in other studies ${ }^{(35,36)}$. Ultimately, improvements in formal education may result in better household dietary diversity via different pathways such as improved choices or increased purchasing power. However, the path between caregivers' education and dietary intakes of their 2-5-year-old child is not necessarily a straightforward one. Although increased years of formal education may be linked with increased awareness of a child's needs and better child care practices, increased formal education may also lead to caregivers working outside home, with a decrease in time spent on child care.

As observed in an earlier analysis ${ }^{(37)}$, the type of incomegeneration activity carried out by caregivers predicted diversity of ASF. An ASF-related business was not more profitable than non-ASF-businesses; however, the former appeared to increase ASF availability for young children. Although the variables introduced to the models were associated with the outcomes, the effect sizes, especially for caregivers' knowledge and attitudes and formal education, were quite modest, suggesting a need to explore other variables as possible determinants of the outcomes.

Several factors may lead to the observed differences in dietary consumption across the agro-ecological zone in Ghana for example, the Coastal Savannah and Guinea Savannah agro-ecological zones are noted for drier climates, increasing rainfall variability and hotter temperatures that often result in decreased food output and incomes ${ }^{(38)}$. The Forest Transitional ecological zone on the other hand has a more favourable climate and serves as an important food hub for the country. Thus, this zone is favoured with increased availability and access to food. Cultural practices and/or socio-economic characteristics, such as educational background, across agro-ecological zones may directly or indirectly influence food choices. The final model (model 3) suggested that improved access to needed foods within one's community influenced all three dependent outcomes. Factors such as food choice and other socio-economic factors known to influence dietary intakes but not accounted for in this study may have contributed to the observed unexplained variance ${ }^{(39,40)}$.

\section{Policy implication}

This study provided a novel examination of nutrition knowledge and feeding attitudes of caregivers who are often overlooked but may influence dietary diversity, especially children's ASF consumption in Ghana. Challenges to scaling up interventions include the absence of a generic solution to nutritional problems because of persisting infrastructural and cultural differences. Thus, there might not be a 'one-size-fits-all approach' in addressing health and nutrition interventions.

The different characteristics of populations found in sub-Saharan countries should remind programmes to be sensitive to cultural norms and other factors that drive caregivers' nutrition knowledge and feeding attitudes. More resources (time and money) are needed to improve formal education and incomes of caregivers. Meanwhile, this study showed that nutrition interventions that address the negative information and misconceptions about food and child feeding practice may help achieve more healthy dietary habits.

Considering the relatively small amounts of ASF that can trigger an improvement in child growth ${ }^{(14,41)}$, actions that improve the likelihood of increased intake, such as improving caregivers' knowledge, is important. The amounts of ASF required to improve child growth are attainable even in rural areas, either through home production of eggs or small animals or, more likely, through markets. The final models shown in Table 3 suggest that nutrition interventions to improve dietary intake that neglect the food environment may achieve limited success. Interventions that seek to improve dietary intake need to target households with low levels of caregiver education and plan educational materials that consider caregivers with low formal educational background ${ }^{(42)}$.

Study limitations. Our results should be considered with the following limitations in mind. First, dietary intakes of the household and children were determined by recall, which is subject to recall bias. Thorough staff training and cross-validation were used to bring the effect of recall bias to a minimum. Second, dietary diversity scores and frequency may not be equated to the quality of diet. Consumption of an expensive food item may limit the consumption of other less-expensive but equally nutritious foods. A household that consumes small portions of several ASF several times in the same week may be at an advantage with respect to nutrient adequacy. This notwithstanding, several studies have suggested that dietary diversity score is a fairly accurate predictor of nutrient adequacy and household food security $\left.{ }^{(43,44}\right)$, thus we consider it a strength of this study. Finally, a cross-sectional survey cannot measure adequately behaviours over time and how changes affect food intake. A longitudinal study would better address the issue of temporality.

\section{Conclusion}

The study established that caregivers' nutrition knowledge and feeding attitudes were significantly associated with both the household diet and the intake of ASF by 2-5-year-old children. This study lends credence to the importance of taking caregivers' nutrition knowledge and attitudes into consideration when formulating programmes or policies of intervention in the feeding of children. Caregivers are often the gatekeepers of the household diet and are given the primary responsibility of selecting, preparing and serving food for their children. For nutrition interventions targeted at improving dietary intakes to be successful, researchers and programme managers need to 
understand better the existing nutrition knowledge and attitudes of their target group - the caregivers of young children.

\section{Acknowledgements}

This work was supported by the Global Livestock Collaborative Research Support Programme of the Office of Agriculture, Bureau for Economic Growth, Agriculture, and Trade, United States Agency for International Development (USAID) under terms of grant no. PCE-G-00-98-0003600. The opinions expressed here are those of the authors and do not necessarily reflect the views of the USAID.

This study falls under a broader study - The Enhancing Child Nutrition through Animal Source Food Management (ENAM) project. G. S. M., E. K. C., O. S.-D., A. L., B. K. A. and L. M. B. were the principal investigators who designed and conducted the ENAM research. A. K. C. was a graduate student under the ENAM project and contributed in preparing questions for investigation, analysed the data and wrote the paper. The authors thank Paul Voss for kindly providing the statistical expertise for the multilevel analysis. All authors read and approved the final version of the manuscript.

The authors declare that there are no conflicts of interest.

\section{References}

1. Fanzo J (2012) The Nutrition Challenge in Sub-Saharan Africa. New York, NY: United Nations Development Programme.

2. Ejide B (2012) Childhood malnutrition in sub-Saharan Africa and sustainable development: the role of corruption, the World Bank, and the west. J Sustainable Dev Afr 14, 32-55.

3. Pelletier D, Frongillo E \& Habicht J-P (1993) Epidemiologic evidence for a potentiating effect of malnutrition on child mortality. Am J Public Health 83, 1130-1133.

4. Lartey A (2013) Nutrition in sub-Saharan Africa: setting the stage for a post-2015 take-off. IDS Bull 44, 49-54.

5. Larson N, Neumark-Sztainer D, Hannan P, et al. (2007) Family meals during adolescence are associated with higher diet quality and healthful meal patterns during young adulthood. J Am Diet Assoc 107, 1502-1510.

6. Orrell-Valente J, Hill L, Brechwald W, et al. (2007) "Just three more bites": an observational analysis of parents' socialization of children's eating at mealtime. Appetite 48, 37-45.

7. Bentley M, Wasser H \& Creed-Kanashiro M (2011) Responsive feeding and child undernutrition in low-and middle-income countries. J Nutr 141, 502-507.

8. Black M \& Aboud F (2011) Responsive feeding is embedded in a theoretical framework of responsive parenting. $J$ Nutr 141, 490-494.

9. Mirmiran P, Azadbakht L \& Azizi F (2006) Dietary diversity within food groups: an indicator of specific nutrient adequacy in Tehranian women. J Am Coll Nutr 25, 354-362.

10. Hsu-Hage B \& Wahlqvist M (1996) Food variety of adult Melbourne Chinese: a case study of a population in transition. World Rev Nutr Diet 79, 53-69.

11. Darapheak C, Takano T, Kizuki M, et al. (2013) Consumption of animal source foods and dietary diversity reduce stunting in children in Cambodia. Int Arch Med 6, 29.

12. Bwibo NO \& Neumann CG (2003) The need for animal source foods by Kenyan Children. J Nutr 133, 3936S-3940S
13. Newmann C \& Harris D (1999) Contribution of Animal Source Foods in Improving Diet Quality for Children in the Developing World. Washington, DC: The World Bank.

14. Long JK, Murphy SP, Weiss RE, et al. (2012) Meat and milk intakes and toddler growth: a comparison feeding intervention of animal-source foods in rural Kenya. Public Health Nutr 15, 1100-1107.

15. Engle P \& Pelto G (2011) Responsive feeding: implications for policy and program implementation. J Nutr 141, 508-511.

16. ICF Macro (2010) Nutrition of Children and Women in Ghana: A New Look at Data from the 2008 Ghana Demographic and Health Survey. Claverton, MD: ICF Macro.

17. Adu-Afarwuah S, Lartey A, Brown KH, et al. (2007) Randomized comparison of 3 types of micronutrient supplements for home fortification of complementary foods in Ghana: effects on growth and motor development. Am J Clin Nutr 86, 412-420.

18. Adu-Afarwuah S, Lartey A, Brown KH, et al. (2008) Home fortification of complementary foods with micronutrient supplements is well accepted and has positive effects on infant iron status in Ghana. Am J Clin Nutr 87, 929-938.

19. Ghana Statistical Service, Noguchi Memorial Institute for Medical Research (NMIMR), \& ORC Macro. MEASURE/DHS + (Programme) (2004) Ghana Demographic and Health Survey, 2003. Calverton, MD: Ghana Statistical Service.

20. Colecraft E, Marquis G, Sakyi-Dawson O, et al. (2012) Planning, design and implementation of the enhancing child nutrition through animal source food management (ENAM) project. Afr J Food Agric Nutr Dev 12, 5687-5708.

21. Marquis GS, Colecraft EK, Sakyi-Dawson O, et al. (2015) An integrated microcredit, entrepreneurial training, and nutrition education intervention is associated with better growth among preschool-aged children in rural Ghana. J Nutr 145, 335-343.

22. Brown K, Dewey K \& Allen L (1998) Complementary Feeding of Young Children in Developing Countries: a Review of Current Scientific Knowledge. WHO/NUT/98.1 228-240. Geneva: WHO.

23. Murphy SP \& Allen LH (2003) Nutritional importance of animal source foods. J Nutr 133, 3932S-3935S.

24. Colecraft E, Marquis GS, Aryeetey R, et al. (2006) Constraints on the use of animal source foods for young children in Ghana: a participatory rapid appraisal approach. Ecol Food Nutr 45, 351-377.

25. Food and Agriculture Organization (2007) Guidelines for measuring household and individual dietary diversity. Rome: FAO, the Food and Nutrition Technical Assistance (FANTA) Project.

26. Kennedy G, Ballard T \& Dop MC (2011) Guidelines for Measuring Household and Individual Dietary Diversity. Rome: FAO.

27. World Food Programme (2008) Food Consumption Analysis. Calculation and Use of the Food Consumption Score in Food Security Analysis, Vulnerability Analysis and Mapping. Rome: United Nations World Food Programme.

28. Hamilton L (2012) Statistics with Stata: version 12. Boston, MA: Cengage Learning.

29. Caswell JA \& Yaktine AL (2013) Supplemental Nutrition Assistance Program: examining the evidence to define benefit adequacy. Washington, DC: National Academies Press.

30. Rabe-Hesketh S \& Skrondal A (2006) Multilevel modelling of complex survey data. $J R$ Stat Soc Ser A 169, 805-827.

31. Hox J (2010) Multilevel Analysis: Techniques and Applications. New York: Routledge.

32. Ehiri JE \& Prowse JM (1999) Child health promotion in developing countries: the case for integration of environmental and social interventions? Health Policy Plan 14, 1-10. 
33. Aidam BA, Pérez-Escamilla R \& Lartey A (2005) Lactation counseling increases exclusive breast-feeding rates in Ghana. J Nutr 135, 1691-1695.

34. Blaylock J, Smallwood D, Kassel K, et al. (1999) Economics, food choices, and nutrition. Food Policy 24, 269-286.

35. Hendricks K, Briefel R, Novak T, et al. (2006) Maternal and child characteristics associated with infant and toddler feeding practices. J Am Diet Assoc 106, Suppl. 1, S135-S148.

36. Christian A, Lartey A, EK C, et al. (2012) Relationship between caregivers' income generation activities and their children's animal source food intake. Afr J Food Agric Nutr Dev 12, 5746-5758.

37. Yaro JA (2013) Building Resilience and Reducing Vulnerability to Climate Change: Implications for Food Security in Ghana. Legon, Ghana: University of Ghana.

38. Pollard J, Kirk S \& Cade J (2002) Factors affecting food choice in relation to fruit and vegetable intake: a review. Nutr Res Rev 15, 373-387.

39. Kell K, Judd S, Pearson K, et al. (2015) Associations between socio-economic status and dietary patterns in US black and white adults. Br J Nutr, 1-8.
40. Neumann CG, Murphy SP, Gewa C, et al. (2007) Meat supplementation improves growth, cognitive, and behavioral outcomes in Kenyan children. J Nutr 137, 1119-1123.

41. Aemro M, Mesele M, Birhanu Z, et al. (2013) Dietary diversity and meal frequency practices among infant and young children aged 6-23 months in Ethiopia: a secondary analysis of Ethiopian Demographic and Health Survey 2011. J Nutr Metab 2013, 782931 .

42. Torheim L, Ouattara F, Diarra M, et al. (2004) Nutrient adequacy and dietary diversity in rural Mali: association and determinants. Eur J Clin Nutr 58, 594-604.

43. Ruel MT (2003) Is dietary diversity an indicator of food security or dietary quality? A review of measurement issues and research needs. Food Nutr Bull 24, 231-232.

44. Steyn N, Nel J, Nantel G, et al. (2006) Food variety and dietary diversity scores in children: are they good indicators of dietary adequacy? Public Health Nutr 9, 644-650. 